\title{
LAS ESPORAS RECIENTES DE LOS MUSCI (BRYOPHYTA) DE LAS TURBERAS DE Sphagnum DE TIERRA DEL FUEGO
}

\author{
Celina M. Matteri ${ }^{1}$
}

\begin{abstract}
Significant variation in moss spore morphology is demonstrated by the observation and description of the spores of 14 moss species which are typical elements of Sphagnum bogs from Tierra del Fuego (Argentina). Spore analysis in the light microscope and in the scanning electron microscope enables easy identification of families, most of the genera and also some species from the recent moss flora. It is suggested that these observations should also allow identification of moss spores from Quaternary peat deposits in the same area where they are expected to occur. Thus, moss spore mor. phology provides information relevant to taxonomic and phylogenetic as well as paleopalynological studies.
\end{abstract}

\section{RESUMEN}

La considerable variación de la morfología de las esporas de los Musci es demonstrada por la observación y descripción de las esporas de 14 especies de musgos que son los elementos más típicos de las turberas de Sphagnum de Tierra del Fuego (Argentina). El análisis de las esporas al microscopio óptico y al microscopio electrónico de barrido, permite distinguir las familias, la mayoría de los géneros y también algunas de las especies de la flora muscológica actual. Estas observaciones sugieren la posibilidad de identificación de esporas de musgos de depósitos turbosos Cuaternarios de la misma área, donde es posible que se encuentren. De este modo, la morfología de las esporas de los Musci provee informaciones relevantes tanto para estudios taxonómicos y filogenéticos como también paleopalinológicos.

\section{INTRODUCCION}

En el proyecto de estudio de la Flora Muscológica de Tierra del Fuego (PID-CONICET 3-071900/85) incorporamos como caracteres de alta significación taxonómica a nivel familiar, genérico y a veces también específico, las ornamentaciones de las esporas de los Musci, observadas al microscopio óptico (MOP) y al microscopio electrónico de barrido (MEB). El conocimien to de la morfología de las esporas de estas plantas en al ámbito regional es bastante escaso. Las contribuciones publicadas (KÜH. NEMANN \& GONCCALVES CARRALVES, 1979; MATTERI, 1980, 1983, 1984) corresponden a estudios palino-taxonómicos aislados, pero que constituyen una clara demonstración de la importancia creciente de la aplicación de la estructura de las esporas en investigaciones taxonómicas y filogenéticas, desestimando al mismo tiempo, la común convicción de la monotonía de las esporas de los Musci (SORSA, 1976). Por otra parte, el reconocimiento de las estructuras esporales de los musgos recientes contribuye naturalmente al mayor desarrollo de los estudios paleopalinológicos en los que, en general, no se encuentran correspondencias con esporas de musgos actuales, tal vez con la única excepción de las del género Sphagnum.

Como primera contribución a la palinología sistemática de los Musci de Tierra del Fuego se han seleccionado para este trabajo los taxa más representativos, en términos de cobertura, de un tipo de vegetacion muy abundante en la región Fueguina, como son las turberas de Sphagnum.

Especificamente, se analizaron materiales de: Turbera de Laguna Negra $\left(54^{\circ} 50^{\prime} \mathrm{S}\right.$, $68^{\circ} 34^{\prime} \mathrm{W}$ ), en Lapataia, situada a ca. $26 \mathrm{~km}$ al SO de Ushuaia, capital del Territorio, y Turbera de Tierra Mayor ( $54^{\circ} 52^{\prime} \mathrm{S}, 67^{\circ} 25 \mathrm{w}$ ), equidistante de la anterior, hacia al NE, de Ushuaia. Ambas turberas son hipoacidófilas según la clasificación de ROIVAINEN (1954), con pH 5,5 - 6,5 y están ubicadas en un área boscosa caducifolia de Nothofagus pumilio.

1 Museo Arg. Cs. Nat. B. Rivadavia y Consejo Nacional de Investigaciones Científicas y Técnicas, Buenos Aires, Argentina. 
Las comunidades de musgos de ambas turberas son bastante similares en su composición (Tabla 1), con dominancia de cuatro taxa turfófilos obligados o frecuentes, de distribución mundial amplia o bipolares y que representan a tres géneros y tres familias diferentes. Entre las especies asociadas de estas ccmunidades, que son turfófilas obligadas y endémicas en su distribución, se analizaron cinco taxa, que pertenecen a cinco géneros y familias distintas. Finalmente, entre las turfofilas ocasionales, o especies de gran amplitud ecológica, se estudiaron cinco taxa adicionales, de distribución generalmente circumsubantártica y que pertenecen a cinco géneros y tres familias.

Se analizó un total de 14 taxa que representan a 13 géneros y a 10 familias de musgos.

\section{MATERIALES Y METODOS}

Los materiales analizados fueron tomados de ejemplares esporulados de colecciones recientes realizadas en el Territorio Nacional de Tierra del Fuego en las turberas mencionadas más arriba y cuyo número de registro se indica en cada descripción ( $\left.\mathrm{CM} \mathrm{n}^{\circ}\right)$. Sólo el material de Sphagnum magellanicum esporulado provie. ne del hemisferio Norte (Finlandia, Regio aboensis, V. Tenhola, Isoviita 20.8 .66 , H). Las esporas maduras fueron secadas al aire y no se sometieron a ningún tratamiento de preparación previo. Para las observaciones al MOP se montaron en agua o en gelatina glicerinada. Las observaciones y microfotografías al MEB fueron realizadas en un microscopio electrónico de barrido JEOL JSM 25 S II, con material metalizado según SAITO \& HIROHAMA (1974). Las dimensiones de los diámetros ecuatorial y polar se obtuvieron de la medición de 50 esporas de un mismo ejemplar. Para la terminologia descriptiva de la forma y estructura de las esporas se siguió a MCCLYMONT (1955), KREMP (1968), BURUS \& JARAI KOMLODI (1975), SORSA (1976), CLARKE (1979) y MOGENSEN (1985). La posición de los polos y áreas aperturales de las esporas están basadas únicamente en las variaciones de la densidad y forma de los procesos y a veces por la presencia de depresiones o fisuras de la pared esporal. Las muestras analizadas están depositadas en la palinoteca del PROIM (BA).

\section{Sphagnum magellanicum Brid.}

$$
\text { Musc. Rec. 2(1): 24, } 1798 \text {, }
$$
plia.
Aspecto microscópico (MOP) - Esporas tetraédricas, heteropolares, radiosimétricas, con una clara marca trilete en el polo proximal, 28 (25-32) $\mu \mathrm{m}$, amarillas, granulosas. Exina $0,7 \mu \mathrm{m}$.

Ornamentación (MEB) - La superficie de la exina es rugosa a ondulada y con depósitos irregulares de gránulos de perina en el polo distal. En el polo proximal se observa una marca trilete definida y la superficie de la espora es densamente granulosa, con algunos gránulos libres $\mathrm{y}$ anastomosados (Figs. 1, 2).

Ecologia - Heliófila, turfófila obligada y dominante en las turberas en estudio, donde la esporulación es rara. Se presenta en superficies onduladas $\mathrm{y}$ algodonosas o en montículos duros y a veces también sumergida en charcas turbosas.

Nota - Esporas generalmente distinguibles en el campo paleopalinológico a nivel genérico. Sin embargo, su fina ornamentación permite también distinguir las especies (TALLIS. 1962; MCQUEEN, 1985). (Material: Isoviita 20.8.66, H).

\section{Sphagnum fimbriatum Wils.}

\section{In Hook., Crypt. Bot. Antarct. Voyage 92. 1845}

\section{Distribución - Bipolar.}

Aspecto microscópico (MOP) - Esporas tetraédricas, definidamente heteropolares, radiosimétricas, con una marca trilete proximal bien distinta, $24(21,5-29) \mu \mathrm{m}$, amarillas, granulosas. Exina 0,5 $\mu \mathrm{m}$ (Fig. 5).

Ornamentación (MEB) - La superficie esporal es densamente rugulosa y con numerosos gránulos de perina en el polo distal. En el polo proximal la superficie es menos rugulosa y los gránulos más escasos, formando un cíngulo en el ecuador y agrupadas en torno a la marca trilete. Los brazos de la marca son largos (ca. $7,5 \mu \mathrm{m}), y$ llegan casi hasta el ecuador de la espora. (Figs. 3, 4).

Ecologia - Semiheliófila, turfófila obligada que esporula frecuentemente en el área Fueguina. Se desarrolla en zonas marginales de las turberas de Sphagnum, sobre superficies planas a menudo asociada con Cyperaceae.

Nota - Las diferencias específicas de las esporas de esta especie con la anterior, riside en su ornamentación mucho menos definida y densa que en $S$. magellanicum. (Material: CM 2314).

\section{Calliergon sarmentasum (Wahlenb.) Kindb.}

Canad. Rec. Sc. 6(2): 72, 1894. 
Paleobot. e Palinol. na América do Sul - 1985 - Bol. IG-USP, Inst. Geociênc., Univ. S. Paulo, 17; 1.147, 1986

Tabla 1 -- Especies de musgos de turberas.

FAMILIA

a. Turfófilas obligadas Sphagnummagellanicum Brid. Sphagnum fimbriatum Wils. Calliergon sarmentosum (Wahlenb.) Kindb.

Sphagnaceae

Sphagnaceae

Amblystegiaceae

b. Turfófila frecuente

Polytrichum juniperinum Hedw. var. affine (Funck) Brid.

Polytrichaceae

\section{ESPECIES ASOCIADAS}

a. Turfófilas obligadas

Skottsbergia paradoxa Card.

Trematodon geniculatus Matteri

Tayloria dubyi Broth.

Neomeesia paludella (Besch.) Deguchi

Distichophyllum fuegianum Matteri

Ditrichaceae

Dicranaceae

Splachnaceae

Maesiaceae

Hookeriaceae

b. Turfófilas ocasionales

Psilopilum trichodon (Hook.f. \& Wils.) Mitt.

Polytrichaceae

Conostomum pentastichum (Brid.) Lindb.

Bartramiaceae

Philonotis vagans (Hook.f. \& Wils.) Mitt.

Bartramiaceae

Bartramia patens Brid.

Tortula robusta Hook. \& Grev.

Bartramiaceae

Pottiaceae

Distribución - Bipolar.

Aspecto microscópico (MOP) - Esporas globosas, con el polo proximal aplanado, 16 (13-20) $\mu \mathrm{m} \times 10,8(7.12) \mu \mathrm{m}$, amarillentas, con densos y gruesos gránulos regularmente distribuídos en toda la superficie. Exina de mertos de $0,3 \mu \mathrm{m}$ de espesor (Fig. 11).

Omamentación (MEB) - Los procesos superficiales del tipo bacula o pila están regularmente espaciados y se presentan libres o anastomosados dejando áreas de la exina lisas. Los procesos son más densos y más pequeños en el polo proximal (Figs. 9, 10).

Ecología - Turfófila obligada, heliófila, que ocasionalmente se halla esporulada. Ocupa áreas considerables en depresiones de las turberas de Sphagnum.

Nota - Descripción e ilustración en KANDA (1976). No se conocen otras observaciones de estas esporas bajo el MEB. Su ornamentación es similar a la de otras especies europeas del mismo género (BOROS \& JARAIKOMLODI, 1975). (Material: CM 3114).

\section{Polytrichum juniperinum Hedw, var. affine}

(Funck) Brid.

Spec. Musc. 1:49, 1806.

Distribución - Bipolar.
Aspecto microscópico (MOP) - Esporas globosas, 11,6 (9.13) $\mu \mathrm{m} \times 10(9.11) \mu \mathrm{m}$, ama. rillentas, finamente granulosas. Exina ca. $0,5 \mu \mathrm{m}$ (Fig. 8).

Ornamentación (MEB) - Toda la superficie esporal presenta procesos muy espaciados y regularmente distribuídos, de tipo cono. Hacia el polo proximal se observa una área apertu$\mathrm{ral}$ en forma de disco deprimido $(2-3 \mu \mathrm{m})$, rodeada por conos más pequeños y más espaciados que en el polo distal. (Figs. 6, 7).

Ecología - Turfófila frecuente en la turberas de Sphagnum y a menudo esporulada. Heliófila obligada, que habita también en suelos desnudos y áreas marginales de bosques caducifolios.

Nota - La ornamentación esporal es similar a la observada en la variedad típica (SCHIAVONE, 1978), con sutiles diferencias en cuanto a la densidad de los procesos. Especie descripta e ilustrada en GREENE (1973, como Polytrichum alpestre Hoppe). (Material: CM 3129).

\section{Skottsbergia paradoxa Card.}

Rev. Bryol. 32:47, 1905

Distribución - Endémica. 

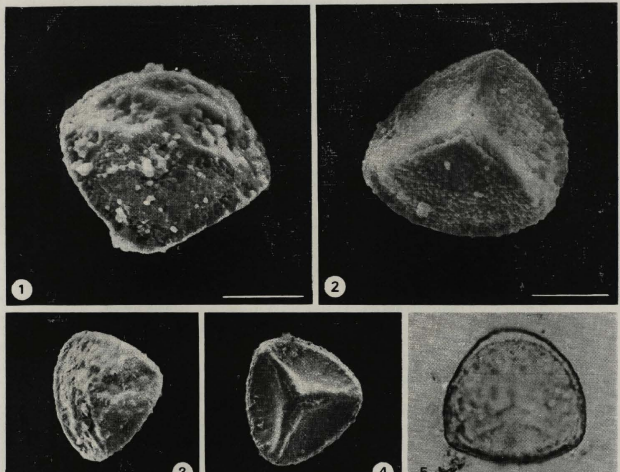

(3)
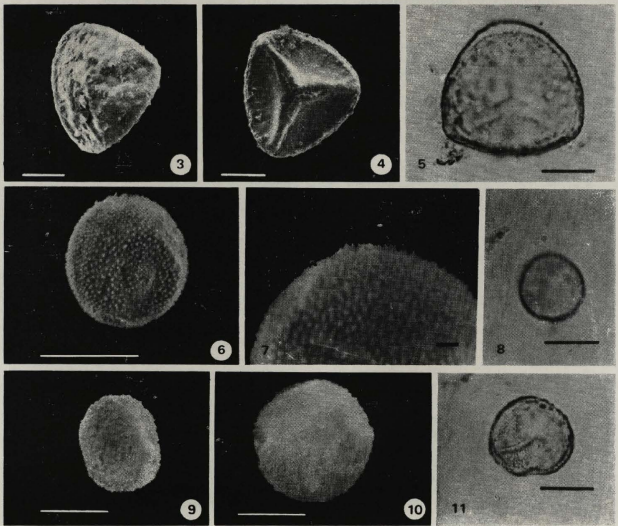

Figuras 1-11 - Microfotografías de esporas. 1, 2. Sohagnum magel/anicum (MEB). 3-5. Sphagnum fimbria tum (3, 4, MEB; 5, MOP). 6-8. Poly trichum juniperinum var. affine $(6,7, \mathrm{MEB} ; 8$, MOP). 9-11. Calliergon sarmentosum $(9,10$, MEB: 11 , MOP). Escalas $10 \mu \mathrm{m}$. 
Aspecto microscópico (MOP) - Esporas esféricas a cóncavo-convexas, $48(45-52,5) \mu \mathrm{m}$ x $37(33.41) \mu \mathrm{m}$, amarillo verdosas, irregularmente granulosas. Exina ca. $2 \mu \mathrm{m}$ (Fig. 20).

Ornamentación (MEB) La superficie esporal es densamente granulosa con procesos de menos de $1 \mu \mathrm{m}$ de diámetro. Una segunda capa de procesos de tipo verruga se distribuye irregularmente sobre los gránulos y también se hallan libres, aislados o agrupados, sobre aquellos. No se observan áreas aperturales definidas (Figs. 18, 19).

Ecologia - Especie asociada en las comunidades de Sphagnum magellanicum, turfófila obligada, semiheliófila. Es una helofita que se presenta frecuentemente esporulada en los bor. des expuestos de canales y charcas de turbera.

Nota - Estas son las primeras ilustraciones microscópicas (MOP y MEB) de las esporas de esta especie y complemento indispensable para evaluar su posición familiar (Material: CM 3119).

\section{Trematodon geniculatus Matteri}

Revta. Mus. Arg. Cs. Nat. B. Rivadavia, Botánica, 5, n? 15:262, 1980 .

Distribución: Endémica.

Aspecto microscópico (MOP) - Esporas subtetraédricas, $32(30-36) \mu \mathrm{m} \times 26(25,5$. 27) $\mu \mathrm{m}$, amarillo parduscas. Son definidamente heteropolares con la superficie irregularmente baculada y un área apertural proximal, más o menos circular. Exina $1 \mu \mathrm{m}$ de espesor sin procesos (Fig. 17).

Ornamentación (MEB) - Bajo el MEB presenta báculos muy densos, aislados o unidos lateralmente en hileras de 2 a 5 . Sobre los báculos se observan gránulos o gemas de forma y tamaño irregulares y caducos, probablemente de perina. En el polo proximal los báculos desaparecen y se observa una superficie circular verru. gosa que rodea el área apertural (Figs. 14, 15, 16).

Ecología - Especie asociada de las comunidades de turbera, que esporula abundantemente, esciófila, acidófila y turfófila obligada. Vive dentro de los canales de desague de las turberas de Sphagnum.

Nota - Especie descripta e ilustrada en MATTERI (1980), siendo estas las primeras observaciones de las esporas bajo el MEB. La comparación de estas observaciones con las de HIROHAMA (1976) sobre otras especies del mismo género, permite adjudicar a la ornamentación de las esporas en éste genero un signifi- cativo valor taxonómico a nivel específico $(\mathrm{Ma}$ terial: CM 3124).

Tayloria dubyi Broth.

Nat. Pfl. 1(3):502, 1903.

Distribución - Endémica.

Aspecto microscópico (MOP) - Esporas cóncavo-convexas, $10,6(8,25-12,75) \mu \mathrm{m} \times 8(5$. 10) $\mu \mathrm{m}$, translúcidas, lisas a débilmente punteadas. Exina menos de $0,3 \mu \mathrm{m}$ de espesor.

Ornamentación (MEB) - Toda la superficie de la exina es densa y regularmente escrobiculada. El polo proximal está definido por una depresión en la superficie cóncaya de la espora, sin claras diferencias ornamentales (Figs. 21, 22).

Ecologia - Turfófila obligada, heliófila, coprófila y profusamente esporulada en las turberas de Sphagnum. Ocurre como especie asociada en los bordes de charcas y lagunas turbosas, sobre excretas animales.

Nota - KOPONEN (1977) señaló la importante significación taxonómica a nivel genérico $\mathrm{y} / \mathrm{o}$ subgenérico de la ornamentación de las esporas de esta familia, correlacionados con otros caracteres sistemáticos. La misma autora (KOPONEN, 1977) demonstró la presencia de perina en toda la ornamentación de otras especies del género Tayloria. (Material: CM 3135).

\section{Neomeesia paludella (Besch.) Deguchi}

Bull. Nat. Sc. Mus, ser. B. (Botany), vol. $9(4): 147,1983$.

\section{Distribución - Endémica.}

Aspecto microscópico (MOP) - Esporas globosas, $21(18-25) \mu \mathrm{m} \times 16,5(14-18) \mu \mathrm{m}$, amarillentas, translúcidas, lisas a ligeramente onduladas. Exina de 1-1,5 $\mu \mathrm{m}$.

Ornamentación (MEB) - Esporas psiladas, de superficie levemente rugosa, y con escasísimos procesos lisos de tipo cono. En el polo proximal se observa un área apertural (?) hundida, rodeada de densas rugosidades concéntricas (Figs. 12, 13).

Ecología - Heliófila, turfófila obligada y asociada en las turberas de Sphagnum. Fructifica sólo ocasionalmente.

Nota - Especie descripta e ilustrada en DEGUCHI (1983), Estas primeras observaciones bajo el MEB de las esporas de la especie complementan la reciente descripción del esporofito (DEGUCHI, 1983) y aportan nuevos elementos de juicio para evaluar su posición familiar. (Material: CM 2269). 

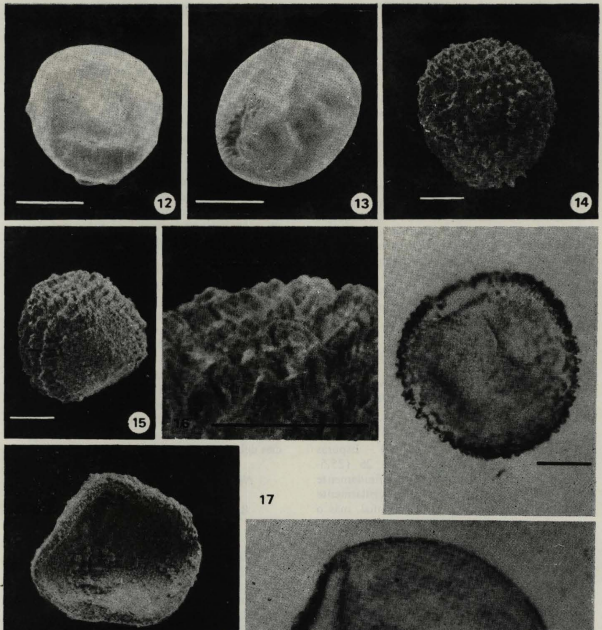

17

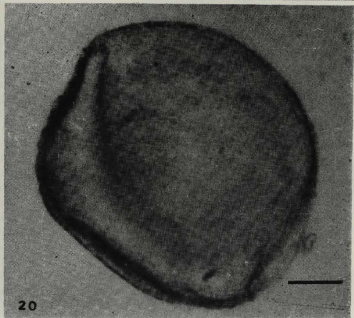

Figuras 12-20 - Microfotografías de esporas, 12, 13. Neomeesia paludella (ME B). 14-17. Trematodon geniculatus (14-16, MEB; 17, MOP). 18-20. Skottsbemia paradoxa (18, 19, MEB; 20, MOP). Escalas 10/tm. 
Paleobot e Paiinol. na America do Sul - 1985 - Bol. IG-USP, Inst. Geociénc., Univ. S. Paulo, 17: 1-147, 1986
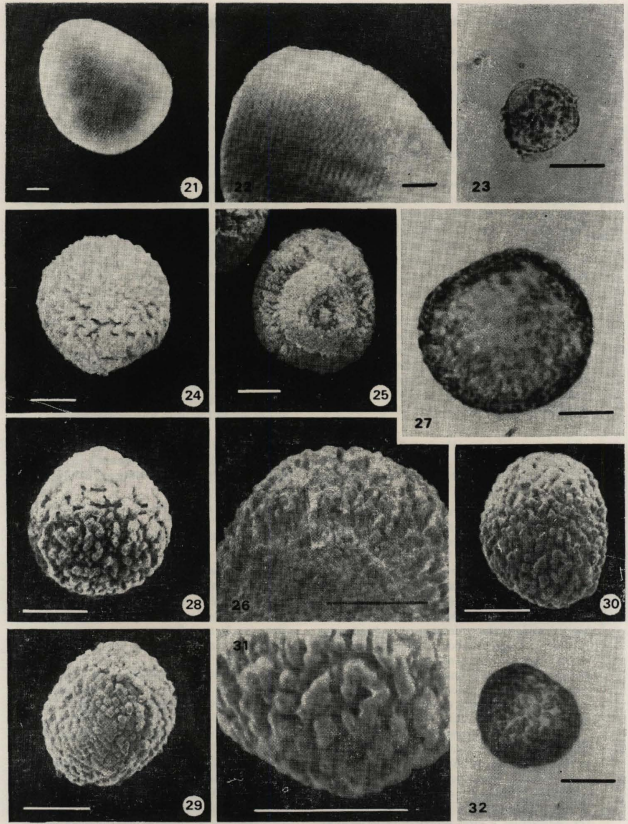

Figuras 21-32 - Microfotografías de esporas. 21, 22. Tayloria dubyi (MEB). 23. Distichophyllum fuegianum (MOP). 24-27. Psilopilum trichodon (24-26, MEB; 27, MOP). 28-32. Philonotis vagans (28-31, MEB; 32, MOP). Escalas $10 \mu \mathrm{m}$. 


\section{Distichophyllum fuegianum (Matteri)}

\section{In S.A. Guarrera, I. Gamundí de Amos y}

D. Rabinovich de Halperin (eds.),

Flora Criptogámica de Tierra del Fuego, tomo XIV (9):20, 1975.

\section{Distribución - Endémíca.}

Aspecto microscópico (MOP) - Esporas globosas, 16,5 (15-18) $\mu \mathrm{m}$, amarillentas, translúcidas, con finos gránulos en toda la superficie esporal. También se observan procesos sueltos y superpuestos. El polo proximal puede interpretarse en la cara aplanada o ligeramente cóncava de la espora. Exina muy delgada (Fig. 23).

Ecología - Turfófila obligada, esciófila. Se presenta ocasionalmente esporulada, dentro de sombríos taludes de canales turbosos, como especie asociada en las comunidades de Sphagnum.

Nota - Descripta e ilustrada em MATTE. RI (1975). Estas esporas aguardam una observación bajo el MEB que en esta oportunidad fue impracticable, y seguramente se separarán de otras especies del mismo género según lo demonstrado en otro trabajo [FIFE \& MATTERI (1984-1985)]. (Material: CM 767).

\section{Psilopilum trichodon (Hook. f. \& Wils.) Mitt.}

\section{J. Linn. Soc. Bot. 12:607, 1869.}

Distribución - Circumsubantártica. Aspecto microscópico (MOP) - Esporas subesféricas con un polo proximal aplanado $y$ distinto, $30(28-33) \mu \mathrm{m} \times 24(22-28) \mu \mathrm{m}$, ama. rillo parduscas, con procesos alargados muy densos y procesos secundarios más pequeños. Exina 1,5 $\mu \mathrm{m}$ de espesor (Fig. 27).

Omamentación (MEB) - La superficie esporal está regular y densamente cubierta por procesos del tipo pila, con procesos secundarios de tipo gema sobre y entre ellos. La ornamen. tación proximal es definidamente diferente con un área apertural discoidal rodeada por un anillo de procesos muy densos del tipo gema o verruga (Figs, 24, 25, 26).

Ecologia - Especie asociada en las turbe. ras de Sphagnum, donde esporula frecuentemente. Heliófila y turfófila ocasional.

Nota - Descripción e ilustración en GREENE (1973). La ornamentación esporal de esta especie permite inferir una convergencia ornamental a nivel genérico, considerando las observaciones de BOROS \& JARAI-KOMLODI (1975) sobre especies europeas de Psilopilum, las de SCHIAVONE (1978) en especies del Norte argentino, y las de SMITH (1974). (Material: CM 3132).

\section{Conostomum pentasticum (Brid.) Lindb}

Oefv. K. Vet, Ak. Foerh. 20:392, 1863 dina

$$
\text { Distribución - Circumsuban tártica - An- }
$$

Aspecto microscópico (MOP) - Esporas reniformes a cóncavo-convexas, $62(50-71) \mu \mathrm{m}$ x 35 (29-38) $\mu \mathrm{m}$, pardo rojizas, verrugosas, con un polo proximal granuloso. Exina ca. $3 \mu \mathrm{m}$ de espesor sin procesos (Fig. 34).

Omamenación (MEB) - La superficie es. poral està densamente cubierta de grandes procesos poligonales del tipo verruga con la superficie granulosa. Sobre ellos se presentan procesos sueltos más pequeños e irregulares. En el polo proximal cóncavo, las verrugas desaparecen individualmente y la textura es contínua y finamente granulosa (Fig. 33).

Ecologia Heliófila, turfófila ocasional, que forma montículos duros en los bordes expuestos de canales turbosos. Es una especie asociada en las turberas de Sphagnum magellanicum donde fructifica sólo ocasionalmente.

Nota - La significación taxonómica a nivel especifico de la ornamentación de las esporas en este género, fue demonstrada en otro tra. bajo (MATTERI, 1984). (Material: CM 3137).

\section{Philonotis vagans (Hook. f. \& Wils.) Mitt.}

$$
\text { J. Linn. Soc. Bot. 4:80, } 1859 .
$$

\section{Distribución - Circumsubantártica.}

Aspecto microscópico (MOP) - Esporas esféricas a ovales con un polo proximal deprimido, 23 (20-28) $\mu \mathrm{m} \times 18$ (16-20) $\mu \mathrm{m}$, pardo rojizas, con la superficie verrugosa con procesos irregulares y anastomosados. Exina $0,7 \cdot 1 \mu \mathrm{m}$ sin procesos (Fig. 32).

Ormamentación (MEB) - La ornamenta. ción esporal es de tipo pilada, con procesos libres o anastomosados y de superficie irregular. Hacia el polo proximal los procesos son más cortos del tipo verruga y más espaciados (Figs. $28,29,30,31$ ).

Ecologia - Especie helofita, ocasionalmente turfófila, heliófila. Vive como especie asociada en las turberas de Sphagnum donde los esporofitos son raros.

Nota - La primeras observaciones bajo el MEB de estas esporas permiten subrayar su va. lor taxonómico a nivel genérico ya señalado en otros trabajos para esta familia (HIROHAMA. 
Paleobot. e Palinol. na América do Sul - 1985 - Bol. IG-USP, Inst. Geociennc., Univ. S. Paulo, 17:1-147, 1986
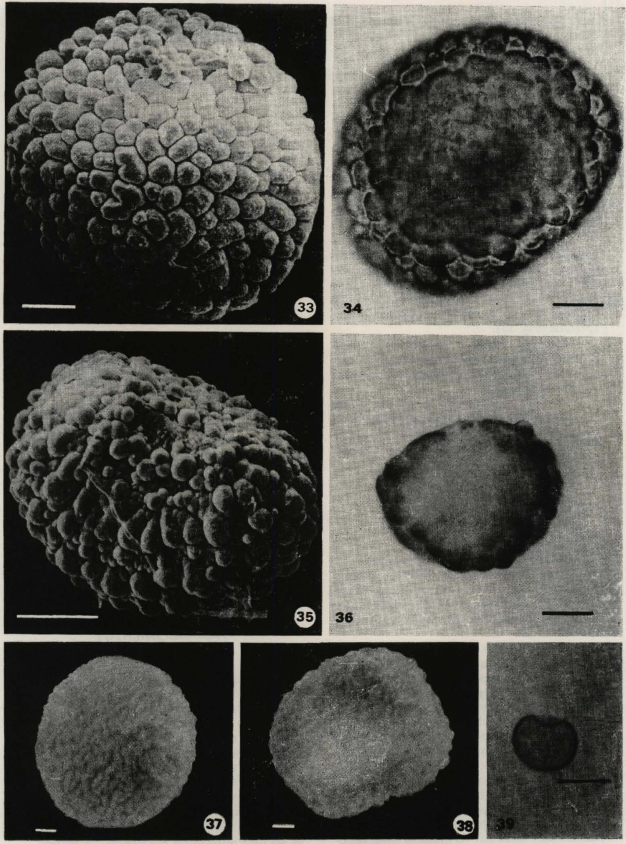

Figuras 33-39 - Microfotografías de esporas. 33, 34. Conostomum pentastichum (33, MEB; 34, MOP). 35. 36. Bartramia patens (35, MEB; 36, MOP). 37-39. Tortula rabusta (37, 38, MEB; 39 , MOP). Escalas $10 \mu \mathrm{m}$. 
1977; MATTERI, 1983, 1984). (Material: CM 2782).

\section{Bartramia patens Brid.}

Musc. Rec. 2(3): 134, 1803 tártica.

Distribución - Circumsubantártica - An-

Aspecto microscópico (MOP) - Esporas reniformes 40 (35-44) $\mu \mathrm{m} \times 30$ (26-34) $\mu \mathrm{m}$, pardo rojizas. Superficie verrugosa con un polo proximal diferenciado. Exina ca. 1,5 $\mu \mathrm{m}$ sin procesos (Fig. 36).

Ornamentación (MEB) - La ornamentación de la espora es denso clavada, con grandes procesos de superficie irregular y procesos menores verrugosos entre aquellos. El polo proxi. mal es diferente por la presencia de un anillo irregular de grandes verrugas rodeando el área apertural (Fig. 35).

Ecologia - Especie asociada en las turberas de Sphagnum donde ocurre frecuentemente esporulada. Heliófila de gran amplitud ecológica y turfófila ocasional.

Nota - Decripción e ilustración en MATTERI (1984). En el mismo trabajo el autor destaca el valor taxonómico genérico de la ornamentación de las esporas en esta familia. (Material: $\mathrm{CM} 3128$ ).

\section{Tortula robusta Hook. \& Grev.}

Edinburgh J. Cs. 1:299, 1824.

\section{Distribución - Circumsubantártica.}

Aspecto microscópico (MOP) - Esporas subesféricas con un polo proximal algo deprimido, $11,8(10-14) \mu \mathrm{m} \times 7,6(6-10) \mu \mathrm{m}$, amari. llentas, finamente papilosas. Exina $0,5 \mu \mathrm{m}$ (Fig. 39).

Ornamentación (MEB) - Esporas densa. mente vermiculadas a verrugosas del polo distal al polo proximal respectivamente. Los procesos son de superficie irregular, pero no se observan definidos procesos secundarios, y sí algunos procesos verrugosos libres. (Figs, 37, 38).

Ecología - Especie heliófila que esporula frecuentemente. Se presenta como especie asociada en las turberas de Sphagnum y también se halla en otros pantanos y en taludes margina. les de bosques caducifolios.

Nota - Descripta e ilustrada en LGHT. OWLERS (1985) sobre material de las Georgias del Sur. Estas son las primeras observaciones de las esporas de esta especie bajo el MEB. Su ornamentación es similar a Tortula norvegi- ca (Web.f.) Wahlenb. (LEWINSKY, 1974) del hemisferio Norte (Material: CM 3136).

\section{CONCLUSIONES}

Como es evidente de los ejemplos presentados en este trabajo la morfologia de las esporas de los Musci es significativamente variada. Aún caracteres tan conspícuos como los observables bajo el microscopio óptico, forma, tamaño, color, y también espesor de la exina, son de interés taxonómico relevante. No obstante, las variaciones en el tipo de ornamentación esporal reveladas por las observaciones bajo el microscopio electrónico de barrido, aumentan considerablemente el valor potencial de estos caracteres en investigaciones tanto taxonómicas como filogenéticas.

La combinación de ambas técnicas de ob. servación de la morfología de las esporas inves. tigadas permite claramente establecer diferencias a nivel específico (Figs. 1.4), a nivel genérico (Figs. 6-8; Figs. 24-27; Figs. 33-36), y obviamente a nivel familiar (Lam. 1 a 4).

Por este motivo, estos caracteres se constituyen en elementos de considerable importancia por sí solos o combinados con otros caracteres, para establecer afinidades entre los taxa como para reevaluar su posición sistemática, definida con caracteres clásicos.

Al mismo tiempo, las esporas de musgos recientes con caracteres taxonómicos reconocibles, especialmente las de mayor diámetro (30-60 $\mu \mathrm{m})$ y con ornamentaciones prominentes, deberian igualmente reconocerse en investi. gaciones paleopalinológicas de depósitos turbosos Cuaternarios.

\section{AGRADECIMIENTOS}

Deseo expresar mi más sincero agradecimiento a mis colegas palinólogos y paleobotánicos quienes estimularon y apoyaron entusiastamente la preparacion de este trabajo con apropiadas críticas y con la provisión de bibliografía esencial. Además, debo un reconocimiento especial al Laboratorio de Palèobotánica del Depto. de Ciencias Biológicas de la FCEN (UA), por la generosa provisión del equipo de microfotografía óptica. El UMIEBFO de la UBA y el SEMEB del CONICET facilitaron la obtención de las microfotografías electrónicas de barrido. La Prof. M.F.G. Carralves colaboró en el montaje de las láminas. 
Paleobot. Palinol. na America do Sul - 1985 - Bol. IG-USP, Inst. Geociênc., Univ. S. Paulo, 17: 1-147, 1986

\section{REFERENCIAS BIBLIOGRAFICAS}

BOROS, A. \& JARAI-KOMLODI, M. (1975) An atlas of recent European moss spores. Budapest, Akadémiai Kiadó, $466 \mathrm{p}$.

CL.ARKE, G.C.S. (1979) Spore morphology and bryophyte systematics. In CLARKE, G.C.S. \& DUCKETT, J.G., eds. Bryophyte Systematics. Systematics Association Special Volume no 14. London and New York, Academic Press, p. 231-250.

DEGUCHI, H. (1983) Neomeesia Deguchi, a new genus of the family Meesiaceae from southern South America. Bull.Natl. Sci. Mus. Ser. B. (Bot.), 9(4):143-148.

FIFE, A.J. \& MATTERI, C.M. [(1984) 1985] Distichophyllum knussei (Lor.) Mitt., a Patagonian moss species newly recorded from New Zealand. Lindbergia 10(3):159-164.

GREENE, S.W. (1973) A synoptic flora of South Georgian mosses: I. Dendroligotrichum, Polytrichum and Psilopilum. Br. Antarct. Surv. Bull. 36:1-32.

HIROHAMA, T. (1976) Spore morphology of bryophytes observed by scanning electron microscope, 1. Dicrana. ceae. Bull. Natl. Sci. Mus. Ser. B (Bot.), 2(2):61-67, pl. 1-5

HIROHAMA, T. (1977) Spore morphology of bryophytes observed by scanning electron microscope, II. Bartramiaceae. Bull. Natl. Sci. Mus. Ser. B (Bot.), 3(1):37-44, pl. 1-6.

KANDA, H. (1976) A revision of the family Amblystegiaceae of Japan Il. J. Sci. Hiroshima Univ., Ser. B, Division 2 (Bot.), 16(1):47-119

KOPONEN, A. (1977) The peristome and spores in Splachnaceae and their evolutionary and systematic significance. Bryophytorum Bibliotheca 13:535-549, pl. I-VIII.

KREMP, G.O.W. (1968) Morphologic encyclopedia of palynology. Contribution no 100. Tueson, Univ. Arizona Press.

KÜHNEMANN, O. \& GONÇALVES CARRALVES, M. (1979) Oedipodium fuegianum Kühn. et Gonç.-Carr. sp. nov. (Musci-Oedipodiaceae). Darwiniana 22(1-3):125-133.

LEWINSKY, J. (1974) An electron microscopical study of the genus Tortula Hedw., with special reference to exine ornamentation. J. Bryol. 8:269-273.

LIGHTOWLERS, P.J. (1985) A synoptic flora of South Georgian mosses: Tortula. Br. Antarct. Surv. Bull. 67:4177.

MATTERI, C.M. (1975) Las Hookeriaceae (Musci) Andino-patagónicas I. Nova Hedwigia 26:649-724.

MATTERI, C.M. (1980) Trematodon geniculatus sp. nov. (Dicranaceae, Musci) de la región Fueguina. Rev. Mus. arg. Cienc. Nat. B. Rivadavia, Botánica, 5(15):261-266.

MATTERI, C.M. (1983) Sobre Bartramia stricta (Bartramiaceae, Musci) en la región Andino-patagónica. Bol. Soc. arg. Bot. 22(1-4):131-141.

MATTERI, C.M. (1984) Sinopsis de las especies Andino-patagónicas, Antárticas y Subantárticas de los géneros Bertramia, Bartramidula y Conostomum (Bartramiaceae, Musci). Darwiniana 25(1-4):143-162.

MoCLYMONT, J.W. (1955) Spore structure in the Musci with special reference to the genus Bruchia. The Bryologist $58: 287-306$.

MoQUEEN, C.B. (1985) Spore morphology of four species of Sphagnum in Section Acutifolla. The Bryologist 88 (1): 1 - 4

MOGENSEN, G.S. (1985) The spore. In SCHUSTER, R.M., ed. New Manual of Bryology, Nichinan. The Hattori Botanical Laboratory, vol 1, chapter 7:325-342.

ROIVAINEN, H. (1954) Studien über die Moore Feuerlands. Ann. Bot.

SAITO, K. \& HIROHAMA, T. (1974) A comparative study of the spores of taxa in the Pottiaceae by use of the scanning electron microscope. J. Hattori Bot. Lab. 38:475-488.

SCHIA VONE, M.M. (1978), Las Polytrichaceae del Noroeste Argentino. Lilloa 35(1):31-65.

SMITH, G.L. (1974) New developments in the taxonomy of Polytrichaceae: Epiphragm structure and spore morphology as generic characters. J. Hattori Bot. Lab. 38:143-150.

SORSA, P. (1976) Spore wall structure in Mniaceae and some adjacent bryophytes. Linnean Soc. Symp. Ser. 1:211229.

TALLIS, J.H. (1962) The identification of Sphagmum spores. Trans. Br. Bryol. Soc. 4:209-213. 